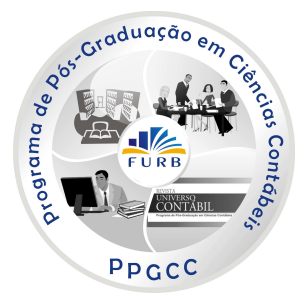

\title{
LA INTEGRACIÓN DEL BALANCED SCORECARD (BSC) Y EL ANALYTIC HIERARCHY PROCESS (AHP) PARA EFECTOS DE JERARQUIZAR MEDIDAS DE DESEMPEÑO Y TOMA DE DECISIÓN EN UNA INSTITUCIÓN FINANCIERA*
}

\author{
INTEGRATING BALANCED SCORECARD (BSC) AND THE ANALYTIC HIERARCHY \\ PROCESS (AHP) TO RANK EFFECTS ON MEASURE OF PERFORMANCE AN \\ DECISION-MAKING IN A FINANCIAL INSTITUTION
}

\author{
Patricia González González \\ Doutora em Controladoria e Contabilidade pela FEA/USP \\ Professora da Universidad Del Valle, Departamento de Contabilidad y Finanzas. \\ Endereço: Calle 4B No. 36-00 - San Fernando - Santiago de Cali - Colombia \\ E-mail pagonza@univalle.edu.co \\ Telefone: 5725185732
}

\section{RESUMEN}

El artículo presenta la metodología que surge de la integración entre el BSC y el AHP, la cual permite calcular los valores relativos en importancia e identificar jerarquías para indicadores y perspectivas. El artículo pretende mostrar como la metodología facilita el cálculo de los índices Globales de Desempeño tanto Reales como Esperados. De tal forma que por medio de ellos, el tomador de decisión puede evaluar el desempeño de la organización, además, de monitorear la implementación de la estrategia. El artículo adopta el estudio de caso para ilustrar la integración entre el BSC y AHP. La compañía caso es el Banco Social Colombiano S.A. de gran trayectoria y reconocimiento en el sector financiero colombiano y cuya visión es la de convertirse en un

\footnotetext{
* Artigo recebido em 13.10.2008. Revisado por pares em 16.12.2008. Reformulado em 04.02.2009. Recomendado em 10.02.2009 por Ilse Maria Beuren (Editora). Publicado em 30.06.2009. Organização responsável pelo periódico: FURB.
} 
establecimiento bancario rentable, siendo el primero en la colocación de créditos de menores cuantías para su nicho de mercado conformado por personas naturales, microempresarios y pequeños empresarios, además de constituirse en un lugar excelente para el desarrollo personal y profesional de sus funcionarios. A través del AHP, los indicadores del tablero de mando se integran con el sistema gerencial enfocado a crear valor para el Banco, de tal forma que un sistema integral de medidas de desempeño se construye con el cálculo de los Índices de Desempeño Esperado y Reales y por medio de este abordaje, el Banco desarrolla la estrategia y monitorea su implementación. El artículo presenta una propuesta metodológica a partir de la cual nos explica como gerenciar indicadores para un Banco a través del uso de una herramienta matemática sin perder de vista la implementación de la estrategia de la organización.

Palabras claves: Cuadro de Mando Integral. Análisis jerárquico de procesos. Indicadores. Mapa Estratégico. Banco. Colombia.

\section{RESUMO}

O artigo apresenta a metodologia que surge da integração entre o BSC e o AHP, a qual permite calcular os valores relativos em importância e identificar hierarquias para indicadores e perspectivas. O artigo pretende mostrar como a metodologia facilita o cálculo dos índices Globais de Desempenho tanto Reais como Esperados. Por meio deles, o tomador de decisão pode avaliar o desempenho da organização, além de monitorar a implementação da estratégia. $\mathrm{O}$ artigo adota estudo de caso para ilustrar a integração do BSC e AHP. A companhia do estudo de caso é o Banco Social Colombiano, de longa trajetória e reconhecimento no setor financeiro colombiano, que pretende se converter em estabelecimento bancário rentável, sendo o primeiro na colocação de créditos de menores quantias para seu mercado alvo, formado por pessoas físicas, microempresários e pequenos empresários, além de constituir-se em um lugar ótimo para o desenvolvimento pessoal e profissional de seus funcionários. Através do AHP, os indicadores do BSC se integram com o sistema gerencial focalizado na criação de valor para o Banco. Um sistema integral de medidas de desempenho se construiu com o cálculo dos Índices de Desempenho Esperados e Reais. Por meio desta abordagem, o Banco desenvolve a estratégia e segue sua implementação. O artigo apresenta uma proposta metodológica a partir da qual explica como gerenciar indicadores para um Banco através do uso de uma ferramenta matemática sem perder o foco na implementação da estratégia da organização.

Palavras-chave: Balanced Scorecard. Analise Hierárquica de Processos. Indicadores. Mapa Estratégico. Banco. Colômbia.

\section{ABSTRACT}

The article presents the methodology that arises from the integration between BSC and the AHP, which allows to calculate the main relative values and to identify hierarchies for indicators and perspectives. Similarly, the paper aims to show how the methodology facilitates the calculation of the Global performance index - both, actual and expected. In such a way that through them the decision makers can evaluate the organization performance, as well as monitor the 
implementation of the strategy. The article adopts case study to illustrate the integration between BSC and the AHP. The case company is Banco Social Colombiano S.A., which has great experience and recognition in the Colombian financial sector and whose vision is to become a profitable bank, being the first to offer small loans for its target market composed of individuals, micro and small entrepreneurs, as well as becoming an excellent place for personal and professional development of its staff. Through the AHP, the indicator panel is integrated with the management, focused on creating value to the Bank. An integral performance measurement system was created with the calculation of actual and expected performance indicators. Through this approach, the Bank develops the strategy and monitors its implementation. The article presents a methodological proposal to explain how to manage Bank indicators, using a mathematical tool, without losing sight of the implementation of the strategy of the organization.

Keywords: Balanced Scorecard. Hierarchy Process Analysis. Indicators. Strategic Map. Bank. Colombia.

\section{INTRODUCCIÓN}

El BSC es una herramienta gerencial creada por Kaplan y Norton, la cual debería ser usada como un sistema de comunicación, información y aprendizaje y, no tanto, como un sistema de control. Este último aspecto generalmente crea confusión en los diferentes usuarios de la herramienta en el momento que se aborda el tema de los indicadores y su número. Más aún cuando Kaplan y Norton (2004) recomiendan un número de 20-25 indicadores.

Los fines relacionados con comunicación, información y aprendizaje que son sugeridos por los creadores del BSC parecen que parcialmente fueran olvidados por los tomadores de decisión, los cuales vienen acostumbrados a tomar decisiones a partir de un número determinado de indicadores financieros.

Esta cultura a veces es difícil de obviar y, por ello, cuando se llega a la etapa de selección de indicadores tanto financieros como no financieros pareciera que todo se centrará en esta tarea y se le diera una gran connotación que desvía la atención de los fines ya mencionados. La labor de identificar y controlar los indicadores involucra a un grupo de personas quienes casi que milimétricamente siguen los cambios que presentan estas medidas. De esa manera se pierde tiempo valioso que debería ser utilizado en la implementación exitosa y monitoreo de la estrategia.

Por lo tanto, el propósito de este artículo es dar respuesta a la siguiente pregunta: De qué manera se puede establecer una forma para priorizar las medidas de desempeño y realizar un continuo seguimiento sin que las actividades de comunicación, información y aprendizaje pierdan su finalidad en el proceso de implementación y ejecución de la estrategia?

Para dar respuesta a esa pregunta, comenzamos el estudio revisando la literatura acerca del Balanced Scorecard (BSC) y del Analytic Hierarchy Process (AHP) para luego pasar al desarrollo del Caso estudio del Banco Social Colombiano S.A en el que se integra el BSC y AHP para efectos de presentar una metodología que nos permite gerenciar indicadores sin perder de vista la implementación de la estrategia. 


\section{REVISIÓN BIBLIOGRAFICA}

\subsection{Balanced Scorecard (BSC)}

Es un paradigma gerencial por medio del cual se pretende traducir la estrategia organizacional en acciones operativas concretas, a través de la integración de las perspectivas de una organización y de los procesos de evaluación.

El modelo establecido por Kaplan y Norton (1997) parte de la premisa que los gerentes deben gerenciar y evaluar sus negocios desde un mínimo de cuatro perspectivas: Financiera, Clientes, Procesos internos y Aprendizaje y Crecimiento. Las cuatro perspectivas llevan a los gerentes a desarrollar e integrar la estrategia entorno a cuatro preguntas:

a) Cómo nos ven los accionistas? (La perspectiva financiera es medida por indicadores como flujo de caja, retorno sobre el patrimonio, participación del mercado, etc.);

b) Cómo los clientes ven la empresa? (La perspectiva de los clientes es medida en parte por indicadores de satisfacción de clientes, entrega a tiempo, retención de clientes, adquisición de clientes, rentabilidad del cliente, participación del mercado, participación en las compras del cliente, etc.);

c) Qué procesos del negocio, la empresa debe mejorar? (La perspectiva de los procesos internos es medida por indicadores tales como ciclos de tiempo, costo unitario, calidad, rotación de inventarios, gastos de investigación y desarrollo, tasas de utilización de mano de obrad, etc.);

d) Puede la empresas continuar innovando y aprendiendo? (La perspectiva de aprendizaje y crecimiento es medida en parte por indicadores tales como cantidad y calidad de la gestión de los empleados, habilidades desarrolladas por los empleados, absentismo, gestión del conocimiento, ratio de información estratégica, número de directivos, etc.)

Del mismo modo, el BSC consta de una estructura que llamaremos tablero de mando y que se compone de los siguientes elementos: objetivos estratégicos, indicadores financieros y no financieros, inductores e iniciativas. El tablero de mando apropiadamente implementado, se convierte en una excelente estructura gerencial que ayuda a los gerentes a monitorear muchos factores que influencian el desempeño. A partir del conjunto de medidas de desempeño, que conforman el BSC, los gerentes pueden saber si la empresa está implementando la estrategia y alcanzando sus objetivos, todo esto en línea con su visión, misión y valores.

\subsection{Priorización de las medidas de desempeño}

Para Kaplan y Norton (1997), una matriz de tablero de mando conformada por los objetivos estratégicos, los indicadores, la meta, los inductores y las iniciativas debe ser usada como sistema de comunicación, información y aprendizaje y, no, como una estructura de control.

El perfil multifuncional que puede transmitir la matriz de tablero de mando a partir de cada uno de los aspectos que la constituyen, pueden llevar a los gerentes a un conflicto $y$, especialmente, en lo que respecta a los indicadores. 
El conflicto aparece cuando los gerentes tienen que lidiar con un número de indicadores que si estimamos en promedio de 4 a 7 por cada perspectiva, para un total de 28 indicadores, aproximadamente. Los gerentes pueden llegar a perder su criterio en cuanto al orden de prioridad e importancia de cada uno de ellos, en la medida que mayor sea su número, ya que, el gerente podrá concluir que todos son importantes.

Esta situación ha llevado a que se pierda por parte de las organizaciones el foco de lo que es el BSC y, es así, como las empresas invierten una gran cantidad de recursos (personas y dinero) y tiempo, exclusivamente, para gerenciar los indicadores. Bajo estas condiciones, la ineficacia de la herramienta aumenta cuando los numerosos indicadores no están interrelacionados.

En estos casos, el problema es mayor porque la información transmitida al tomador de decisión no es la mejor a causa de la falta de interrelación entre ellos pudiendo, inclusive, inducir a malas decisiones. No obstante, Kaplan y Norton (2004) consideran que el número de indicadores no debe ser problema siempre y cuando estén interrelacionados y focalizados en el logro de la estrategia planteada.

Por otro lado, teóricamente numerosos indicadores interrelacionados pueden resultar difíciles de gerenciar e interpretar, ya que estudios relacionados con el procesamiento de información y toma de decisión así, lo han demostrado. Ante una gran cantidad de información, el análisis de la misma resulta difícil para las personas (BADDELEY, 1994).

Hipotéticamente, si, el BSC presenta una cantidad de indicadores que son repartidos en las diferentes perspectivas, pero en cada una de ellas se colocan pocos indicadores, de tal forma que la herramienta estaría permitiendo presentar los indicadores que son más importantes. Los gerentes, sin contar con información adicional, podrían suponer que sí, los indicadores están relacionados y corresponden a un objetivo estratégico primario, entonces, son igualmente importantes.

Sin embargo, el tablero de mando es un formato y la interpretación que se estaría dando no necesariamente sería la correcta para todos los gerentes de diferentes negocios y situaciones estratégicas. Por otro lado hay que adicionar el aspecto de temporalidad que pueden tener los indicadores a través del ciclo de vida del negocio que evidentemente marcarán diferentes prioridades en el uso de ellos, dependiendo de la fase en la que se encuentre la empresa, por ejemplo, de crecimiento, sostenimiento o cosecha.

Para Reisinger, Cravens y Tell (2003), la priorización de las medidas provee un medio para evaluar los cambios en el nivel de importancia de los indicadores a través del tiempo sin que exista la necesidad de crear nuevos indicadores.

Slater, Olson y Reddy (1997) también sugieren la necesidad de priorizar la variedad de indicadores de un tablero de mando. En ese sentido, los autores citados determinan que la estrategia de la empresa puede obligar a desarrollar un tablero de mando que considera las cuatro perspectivas, pero existen medidas adicionales para algunas perspectivas que enfatizan en áreas claves según la estrategia de la compañía.

Para Reisinger, Cravens y Tell (2003) existen dos maneras para priorizar medidas en el BSC. La primera opción consiste en priorizar medidas dentro de cada perspectiva de forma separada. La desventaja de esta opción radica en que es ignorada la importancia relativa de las perspectivas. La segunda opción consiste en colocar pesos en todos los indicadores de tal modo que se pueda realizar una comparación. El hecho de colocar pesos a todos los indicadores está demostrando la prioridad que se le está dando a cada uno de ellos, ya que el peso esta correlacionado con el grado de importancia que le es dado por los miembros de la empresa que 
determinan los pesos y adicionalmente se puede determinar el peso de cada perspectiva. Según los autores citados, la segunda opción es la mejor, en consideración a que permite calcular tanto el valor relativo de importancia de las perspectivas como de los indicadores.

\subsection{Analytic Hierarchy Process (AHP) para efectos de priorizacion}

Una herramienta que se puede utilizar para priorizar los indicadores dentro de un tablero de mando integral es el Analytic Hierarchy Process (AHP).

El AHP fue creado por el matemático Thomas Saaty en la década de los años setenta. El objetivo principal de la metodología es la de ayudar a resolver problemas de toma de decisión donde se involucran varias variables o alternativas. En síntesis podemos decir que la metodología como tal toma una situación compleja, la divide en sus partes (variables) para luego organizarlas en orden de jerarquías. De este modo valores numéricos son asignados a juicios de carácter subjetivo relacionados con la importancia relativa de cada variable. El AHP incorpora valores personales y juicios de una manera lógica. Por lo tanto, la estructura de las jerarquías de un problema dependerá de la imaginación, la intuición y el conocimiento. Por otro lado, los juicios para establecer las jerarquías relativas de las variables dependen de la lógica, la intuición y la experiencia.

Según Fletcher (apud REISINGER, CRAVENS y TELL, 2003), una característica del AHP es la de proveer una estructura que permite la toma de decisión a un grupo, al imponer una disciplina para los procesos de pensamiento a los integrantes del mismo. Esta situación lleva a que se convierta en una herramienta importante en los procesos de planeación estratégica ya que los juicios y las ideas pueden ser cuestionados y, por ende, fortalecidos o debilitados por la evidencia suministrada por las personas que forman el grupo decisorio.

Al existir un consenso en el grupo que toma la decisión, mejora la consistencia del juicio de los gerentes y aumenta la validez y la confiabilidad del AHP como herramienta para toma de decisión. Por lo tanto, un tomador de decisión puede considerar en su análisis, veintiocho (28) indicadores, pero con la ayuda del AHP, las notas dadas por diversos tomadores de decisión a cada indicador son reunidas para crear puntajes, los cuales determinan la prioridad de cada uno. Ya que entre mayor sea el puntaje obtenido, mayor prioridad tendrá ese indicador con respecto a otros.

Para Calantone, Di Benedetto y Smith (1999), el AHP provee mecanismos que reconcilian las inconsistencias que se pueden derivar de los procesos de juicio gerencial. El AHP permite la realización de comparaciones a partir de combinación de parejas de indicadores. Así, el tomador de decisión necesita considerar dos indicadores en el tiempo. Una escala numérica es usada en el proceso de comparación. De tal forma que la información de cada conjunto de comparaciones puede ser convertida en puntajes que permiten crear rangos o clasificaciones para los indicadores. Cabe anotar que dichos rangos o clasificaciones reflejan el juicio de los tomadores de decisión.

La metodología de AHP propuesta por SAATY, en términos generales, consta de cinco pasos:

a) La Construcción de las Prioridades: consiste en la construcción de un conjunto homogéneo de elementos con un elemento individual que representa un propósito global. Llevando esta situación al tablero de mando integral podríamos considerar que la estrategia de la empresa es el propósito global del cual se habla en este paso, como también es la primera prioridad identificada, luego vienen prioridades posteriores 
conformadas por los objetivos específicos que se encuentran en cada una de las cuatro perspectivas;

b) La identificación de parejas: dentro del contexto del tablero de mando integral consiste en comparar dos indicadores dentro de una perspectiva (por ejemplo, comparar dos indicadores dentro de la perspectiva de los clientes) contra un criterio dado para establecer un peso relativo. En este proceso también participan las perspectivas que son comparadas en parejas. Saaty (apud REISINGER, CRAVENS y TELL, 2003, p. 432) anota que la comparación puede ser realizada en términos de importancia, probabilidad o preferencia. De ese modo, los indicadores del tablero de mando integral pueden ser comparados teniendo en consideración la importancia que tienen en el logro del objetivo estratégico o de un buen desempeño financiero;

c) La Síntesis: en este paso se combina matemáticamente todos los valores de preferencia dado a los indicadores de tal forma que se pueda determinar las prioridades. Esto se logra a partir de los valores obtenidos en el paso dos. Los resultados que se obtienen corresponden a los valores relativos de importancia (VRI) y a las jerarquías. Ajustando los resultados para una solución normalizada se garantiza que la suma total de los valores relativos en importancia sean iguales a 1 ó 100\%, para cada perspectiva (financiera, clientes, etc);

d) Revisar las consistencias: para lo cual se debe calcular un porcentaje que se considera como el esperado y otro que es el real. La confrontación de ambos permite determinar la calidad de las decisiones tomadas;

e) Evaluar los Resultados: una vez obtenidos los índices de desempeño esperado y los reales, las prioridades son finalmente evaluadas e interpretadas.

El AHP puede ser usado en una variedad de situaciones a través de diversos ambientes en los cuales se puedan tomar decisiones. Así, por ejemplo, Wind (apud REISINGER, CRAVENS y TELL, 2003) ilustra como esta metodología fue empleada para evaluar diversas estrategias de mercadeo en términos de la importancia relativa de varios componentes de una mezcla de mercados.

Calantone, Di Benedetto y Smith (1999) utilizaron el AHP para determinar prioridades entre un conjunto de nuevos productos disponibles a desarrollar. Min, Mintra y Oswald (1997) aplicaron AHP para comparar aspectos competitivos de benchmarking entre instituciones de salud.

En todos los casos mencionados, al igual que en un escenario de tablero de mando integral, el ambiente es similar, pues en todos ellos el tomador de decisión esta intentando priorizar de la manera más objetiva y coherente los diversos indicadores de éxito o desempeño entre un conjunto de medidas potenciales. Finalmente, la metodología propuesta por Saaty, se puede aplicar de manera genérica para cualquiera que sea el ambiente de toma de decisión.

\section{INTEGRACIÓN DEL BSC Y AHP PARA EL CASO OBJETO DE ESTUDIO - BANCO SOCIAL COLOMBIANO S.A.}

Para ilustrar la aplicación del AHP en el desarrollo de un tablero de mando integral y, específicamente, en lo referente al punto de los indicadores, su jerarquización y al calculo de un índice para monitorear el desempeño global de la organización; trabajaremos con el caso del Banco Social Colombiano S.A. (nombre hipotético). El Banco se encuentra en el proceso de 
monitoreo y evaluación de su desempeño, previo, el establecimiento de unos objetivos estratégicos que surgieron del plan estratégico del Banco.

El Banco Social Colombiano S.A. desde hace más de 90 años, trabaja apoyando el progreso de las personas naturales, los microempresarios y pequeños empresarios, contribuyendo con el desarrollo social del país. Dicha actividad bancaria que tuvo sus orígenes en la fundación de la Caja de Ahorros del Círculo de obreros en Bogotá en 1.911, ha fomentado y valorizado desde entonces el ahorro de los colombianos, hasta constituirse en una institución financiera de perfil popular. Hoy esa entidad, se encuentra entre los Bancos más reconocidos del país.

Actualmente, el Banco atiende a más de un millón de clientes, a través de sus 122 oficinas en 42 ciudades del país. El Banco es una entidad financiera dinámica y creciente con un modelo exitoso de banca social auto sostenible de fácil acceso para las personas, pionero en la colocación de créditos de menores cuantías. El sector microempresarial es uno de los segmentos prioritarios de atención, el apoyo que el Banco a través de la red Banco Social ha prestado al desarrollo y los logros de este sector son motivo de reconocimiento no solo a nivel nacional sino internacional.

El Banco trabaja año tras año en la acertada adecuación de los productos y servicios que ofrece, según las características de los clientes como: montos mínimos exigidos para aperturas de cuentas, agilidad en las transacciones, facilidad de acceso al crédito y productos de fácil manejo. El plan estratégico del Banco, articulado con su visión, fue la de constituirse en un establecimiento bancario rentable, pionero en la colocación de créditos de menores cuantías para su nicho de mercado constituido por personas naturales, microempresarios y pequeños empresarios, además de convertirse en un excelente lugar para el desarrollo personal y profesional de sus funcionarios.

El Banco actualmente identifica un plan estratégico cuyos temas estratégicos son el crecimiento de los ingresos; productividad; soluciones completas para los clientes; relación con los clientes; excelencia operacional; gerenciar las soluciones totales para los clientes; gestión del cliente; innovación; control del riesgo y establecer habilidades para el control del riesgo tal, como se observa en el mapa estratégico exhibido en la Figura 1. 


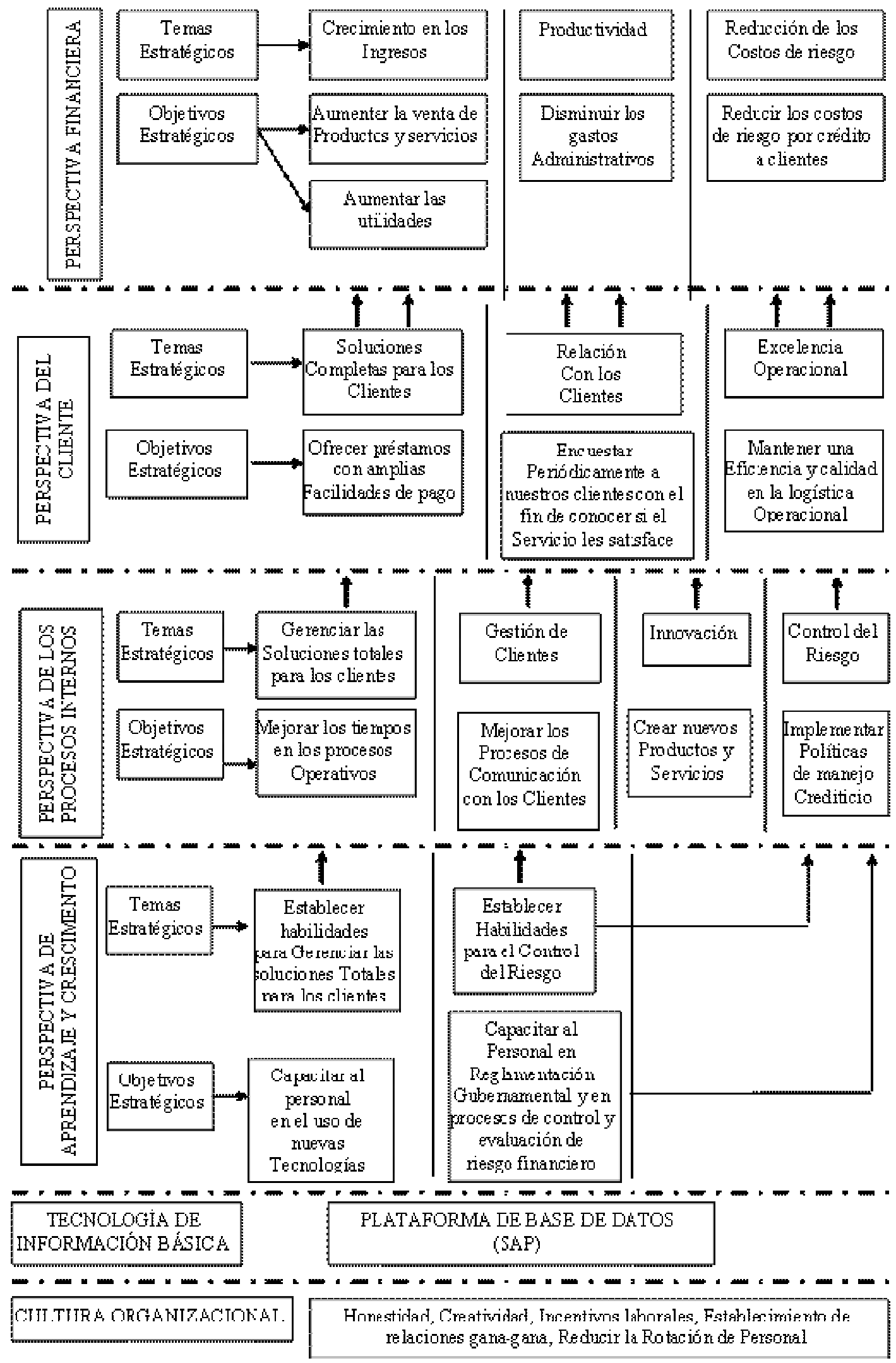

Revista Universo Contábil, ISSN 1809-3337, FURB, Blumenau, v. 5, n. 2, p. 87-105, abr./jun. 2009. 
El AHP fue usado por el equipo que trabajó en el diseño del tablero de mando para priorizar la importancia relativa de cada uno de los objetivos estratégicos que se desprenden de los temas estratégicos ya, mencionados y, para calcular un índice global que facilite el monitoreo del desempeño organizacional. A partir de la adaptación que realizó el grupo del Banco de la metodología planteada por Saaty, pasamos a describir los pasos que fueron seguidos, así:

\section{Paso 1: Establecer la relación entre la meta fijada en la visión, los objetivos estratégicos y la identificación de los indicadores}

El primer paso consiste en identificar los objetivos estratégicos para cada tema estratégico teniendo en consideración las siguientes metas fijadas en la visión: rentabilidad, ser el Banco pionero en la colocación de créditos de menores cuantías para el nicho de mercado identificado y convertirse en un lugar excelente para trabajar.

Una vez realizado este proceso se procede a identificar los indicadores o unidades de medida para cada objetivo estratégico en cada una de las perspectivas a las cuales corresponden (financiera, clientes, procesos internos, aprendizaje y crecimiento). La identificación de cada medida dentro de cada una de las perspectivas es una manera de garantizar que cada perspectiva ha sido tomada en consideración en el desarrollo estratégico y en los procesos gerenciales.

\section{Paso 2: Usar el AHP para determinar los pesos y la importancia relativa de los indicadores}

Para calcular la importancia relativa y los pesos de los indicadores seleccionados para cada objetivo estratégico en cada perspectiva se requiere elaborar la Matriz de Preferencia. La Matriz de Preferencia debe ser elaborada para cada perspectiva en función del conjunto de indicadores identificados. Para elaborar la Matriz es necesario comparar cada par de medidas y colocarles un valor conforme la siguiente escala:

Comparando la medida i y la medida $\mathrm{j}$ :

a) la medida i y j son de igual importancia;

b) la medida i es moderadamente igual a j;

c) la medida i es moderadamente más preferida que $\mathrm{j}$;

d) la medida i es moderadamente más importante que j;

e) la medida i es más importante que j;

f) la medida i está entre importante y supremamente importante que $\mathrm{j}$;

g) la medida i es supremamente importante;

h) la medida i está entre supremamente y absolutamente más importante que j;

i) la medida i es absolutamente más importante que $\mathrm{j}$

Los valores b, $\mathrm{d}, \mathrm{f}, \mathrm{h}$ de la escala corresponden a valores intermedios. 
Tabla 1 - Indicadores identificadas en el tablero de mando de lo Banco Social Colombiano S.A.

\begin{tabular}{|c|c|c|c|c|c|c|c|c|c|}
\hline PERSPECTIVA & OBJETIVO & INDICADOR & BUENO & PESIMO & $\begin{array}{l}\text { REGU } \\
\text {-LAR }\end{array}$ & REAL & META & IDR & IDE \\
\hline \multirow{4}{*}{ Financiera } & \begin{tabular}{|lcc} 
Aumentar ventas & $\mathrm{de}$ \\
productos y servicios & \\
\end{tabular} & Margen de ventas & $25 \%$ & $15 \%$ & $\begin{array}{r}24,99 \\
\%\end{array}$ & $23 \%$ & $25 \%$ & $7,90 \%$ & $8,59 \%$ \\
\hline & Aumentar la utilidades & Margen neta & $15 \%$ & $10 \%$ & $15 \%$ & $14 \%$ & $15 \%$ & $4,58 \%$ & $4,91 \%$ \\
\hline & $\begin{array}{l}\text { Reducir costos por riesgos } \\
\text { en créditos }\end{array}$ & $\begin{array}{ll}\text { Razón } & \text { provis-cartera } \\
\text { CCC } & \\
\end{array}$ & $90 \%$ & $85 \%$ & $\begin{array}{r}89,99 \\
\% \\
\end{array}$ & $86 \%$ & $90 \%$ & $2,17 \%$ & $2,30 \%$ \\
\hline & $\begin{array}{|ll|}\text { Disminuir } & \text { gastos } \\
\text { administrativos } & \\
\end{array}$ & \begin{tabular}{|l|l|} 
Razón gastos \\
administrativos/Utilidad \\
operacional
\end{tabular} & $30 \%$ & $40 \%$ & $\begin{array}{r}30,01 \\
\%\end{array}$ & $45 \%$ & $30 \%$ & $0,53 \%$ & $1,07 \%$ \\
\hline \multirow{3}{*}{ Clientes } & \begin{tabular}{|llr} 
Ofrecer & prestamos & con \\
amplias & facilidades & de \\
pago & & \\
\end{tabular} & $\begin{array}{ll}\text { No. de } & \text { prestamos } \\
\text { otorgados } & \end{array}$ & 100 & 80 & 99 & 105 & 100 & $27,24 \%$ & $\begin{array}{r}25,94 \\
\%\end{array}$ \\
\hline & $\begin{array}{l}\text { Encuestar periódicamente } \\
\text { a los clientes con el fin de } \\
\text { medir satisfacción }\end{array}$ & $\begin{array}{l}\text { \% de encuestas contestadas } \\
\text { favorablemente }\end{array}$ & $90 \%$ & $60 \%$ & $\begin{array}{r}89,99 \\
\%\end{array}$ & $80 \%$ & $90 \%$ & $12,83 \%$ & $14,43 \%$ \\
\hline & $\begin{array}{l}\text { Mantener una eficiencia y } \\
\text { calidad en la logística } \\
\text { operacional en atención a } \\
\text { clientes }\end{array}$ & No. de errores cometidos & 5 & 11 & 6 & 15 & 5 & $-5,92 \%$ & $5,92 \%$ \\
\hline \multirow{4}{*}{$\begin{array}{l}\text { Procesos } \\
\text { internos }\end{array}$} & $\begin{array}{l}\text { Mejorar los tiempos en los } \\
\text { procesos operativos }\end{array}$ & $\begin{array}{l}\text { Minutos gastados en el } \\
\text { análisis de una solicitud }\end{array}$ & 60 & 91 & 60,01 & 55 & 60 & $2,23 \%$ & $2,07 \%$ \\
\hline & $\begin{array}{|lll|}\begin{array}{l}\text { Mejorar los } \\
\text { comunicación }\end{array} & \text { con } & \text { los } \\
\text { clientes } & & \\
\end{array}$ & $\begin{array}{l}\% \text { de clientes llamados en } \\
\text { un período }\end{array}$ & $90 \%$ & $70 \%$ & $\begin{array}{r}89,99 \\
\%\end{array}$ & $60 \%$ & $90 \%$ & $2,31 \%$ & 3,46 \\
\hline & $\begin{array}{l}\text { Crear nuevos productos o } \\
\text { servicios }\end{array}$ & $\begin{array}{l}\text { No. de nuevos productos e } \\
\text { servicios }\end{array}$ & 5 & 2 & 4 & 3 & 5 & $1,09 \%$ & $1,82 \%$ \\
\hline & $\begin{array}{l}\text { Implementar políticas de } \\
\text { manejo crediticio }\end{array}$ & \begin{tabular}{|lcc}
$\begin{array}{l}\text { No. de } \\
\text { implementadas }\end{array}$ & políticas \\
\end{tabular} & 10 & 4 & 9 & 5 & 10 & $1,12 \%$ & $2,24 \%$ \\
\hline \multirow[t]{3}{*}{$\begin{array}{l}\text { Perspectiva } \\
\text { Aprendizaje } \\
\text { crecimiento } \\
\end{array}$} & $\begin{array}{l}\text { Capacitación en nuevas } \\
\text { tecnologías }\end{array}$ & 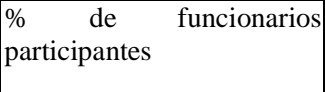 & $80 \%$ & $60 \%$ & $\begin{array}{r}79,99 \\
\%\end{array}$ & $85 \%$ & $80 \%$ & $19,26 \%$ & $18,12 \%$ \\
\hline & \begin{tabular}{|lrr} 
Capacitación en & la \\
ejecución de los & procesos \\
internos & y \\
reglamentaciones & \\
\end{tabular} & $\begin{array}{l}\text { No. de capacitaciones en el } \\
\text { período }\end{array}$ & 10 & 4 & 9 & & 10 & $1,89 \%$ & $5,30 \%$ \\
\hline & $\begin{array}{|llll|}\begin{array}{l}\text { Reducir } \\
\text { personal }\end{array} & \text { rotación de } \\
\end{array}$ & $\%$ de despidos por período & $5 \%$ & $10,99 \%$ & $5,01 \%$ & $2 \%$ & $5 \%$ & $4,53 \%$ & $2,83 \%$ \\
\hline \multicolumn{8}{|l|}{ Total } & $81,78 \%$ & $100 \%$ \\
\hline
\end{tabular}

Al realizar las comparaciones entre los valores ubicados en la fila (i) y en la columna (j), se pueden dar tres tipos de resultado. Para explicar este caso estamos trabajando con los indicadores identificadas en el tablero de mando (Tabla 1) para la perspectiva financiera.

Tabla 2 - Matriz de Preferencia - Medidas de Desempeño: Perspectiva Financiera

\begin{tabular}{|c|c|c|c|c|c|}
\hline Columnas & $\mathbf{j}$ & $\mathbf{j}$ & $\mathbf{j}$ & $\mathbf{j}$ & Filas \\
\hline & $\begin{array}{c}\text { Margen de } \\
\text { Ventas }\end{array}$ & Margen Neta & PC/CC & GA / UO & \\
\hline Margen de ventas & & & & & $\mathrm{i}$ \\
\hline Margen Neta & & & & & $\mathrm{i}$ \\
\hline PC/CC* & & & & & $\mathrm{i}$ \\
\hline GA/UO** & & & & & $\mathrm{i}$ \\
\hline
\end{tabular}

* $\mathrm{PC}=$ Provisión de Cartera; $\mathrm{CC}=$ Cuentas por Cobrar

** GA = Gasto Administración; UO = Utilidad Operacional

El conjunto aij $=1$ ya que $\mathrm{i}=\mathrm{j}$.

En este caso la medida que aparece en la fila es exactamente la misma de la columna. 
LA INTEGRACIÓN DEL BALANCED SCORECARD (BSC) Y EL ANALYTIC HIERARCHY PROCESS (AHP) 98 PARA EFECTOS DE JERARQUIZAR MEDIDAS DE DESEMPEÑO Y TOMA DE DECISIÓN EN UNA INSTITUCIÓN FINANCIERA

Tabla 3 - Matriz de Preferencia - Medidas de Desempeño: Perspectiva Financiera

\begin{tabular}{|c|c|c|c|c|c|}
\hline Columnas & $\mathbf{j}$ & $\mathbf{j}$ & $\mathbf{j}$ & $\mathbf{j}$ & Filas \\
\hline & $\begin{array}{c}\text { Margen de } \\
\text { Ventas }\end{array}$ & Margen Neta & PC/CC & GA / UO & \\
\hline $\begin{array}{c}\text { Margen de } \\
\text { ventas }\end{array}$ & $\mathbf{4}$ & & & & $\mathrm{i}$ \\
\hline Margen Neta & & & & & $\mathrm{i}$ \\
\hline PC/CC & & & & & $\mathrm{i}$ \\
\hline GA/UO & & & & & $\mathrm{i}$ \\
\hline
\end{tabular}

Así, por ejemplo: cuando comparamos la pareja de medidas Margen de Ventas ubicada en la fila i con el Margen de Ventas ubicada en la columna j, el valor a colocar en la matriz será 1. Esto en cumplimiento al primer postulado de la escala.

la medida i y j son de igual importancia.

Tabla 4 - Matriz de Preferencia - Medidas de Desempeño: Perspectiva Financiera

\begin{tabular}{|c|c|c|c|c|c|}
\hline Columnas & $\mathbf{J}$ & $\mathbf{J}$ & $\mathbf{j}$ & $\mathbf{j}$ & Filas \\
\hline Margen de ventas & Margen de Ventas & Margen Neta & PC/CC & GA / UO & \\
& $(1$ & & & & \\
\hline Margen Neta & & & & & \\
\hline PC/CC & & & & & $\mathrm{i}$ \\
\hline GA/UO & & & & & $\mathrm{i}$ \\
\hline
\end{tabular}

El conjunto aij $=\mathrm{k}$ (i es más importante que $\mathrm{j}$ )

Siguiendo con el ejemplo, si al comparar el Margen de Ventas (ubicado en la fila i) con la Margen Neta (ubicada en la columna j), el grupo que está trabajando en determinar el grado de importancia decide que el Margen de Ventas es más importante que el Margen Neto ( o sea, i es más importante que $\mathrm{j}$, en cualquiera de sus niveles) entonces, conforme la escala de notas, a esta premisa le corresponde la nota cinco (5), la cual se coloca en la matriz tal como aparece en la Tabla 5 .

la medida i es más importante que $\mathrm{j}$

Tabla 5 - Matriz de Preferencia - Medidas de Desempeño: Perspectiva Financiera

\begin{tabular}{|c|c|c|c|c|c|}
\hline Columnas & $\mathbf{J}$ & $\mathbf{j}$ & $\mathbf{J}$ & $\mathbf{j}$ & Filas \\
\hline & $\begin{array}{c}\text { Margen de } \\
\text { Ventas }\end{array}$ & Margen Neta & PC/CC & GA / UO & \\
\hline $\begin{array}{c}\text { Margen de } \\
\text { Ventas }\end{array}$ & & $(5$ & & & $\mathrm{i}$ \\
\hline Margen Neta & & & & & $\mathrm{i}$ \\
\hline PC/CC & & & & & $\mathrm{I}$ \\
\hline GA/UO & & & & & $\mathrm{I}$ \\
\hline
\end{tabular}


El conjunto aji $=1 / \mathrm{k}(\mathrm{j}$ es más importante que $\mathrm{i})$

Al comparar la medida de Margen Neta ubicada en la fila i con el Margen de Ventas ubicado en la columna $\mathrm{j}$ (como esta relación es inversa a la presentada en el caso anterior) y, siendo consistentes, el grupo de evaluadores diría que el Margen de Ventas es más importante que el Margen Neta (la columna j es más importante que la fila i) y, por lo tanto, la nota colocada en la matriz sería el inverso de 5 , o sea, $1 / 5=1 / k$.

Tabla 6 - Matriz de Preferencia - Medidas de Desempeño: Perspectiva Financiera

\begin{tabular}{|c|c|c|c|c|c|}
\hline Columnas & $\mathbf{j}$ & $\mathbf{j}$ & $\mathbf{j}$ & $\mathbf{j}$ & Filas \\
\hline & $\begin{array}{c}\text { Margen de } \\
\text { Ventas }\end{array}$ & Margen Neta & PC/CC & GA / UO & \\
\hline Margen de ventas & & & & \\
\hline Margen Neta & (1/5 & & & & i \\
\hline PC/CC & & & & & i \\
\hline GA/UO & & & & \\
\hline
\end{tabular}

Por consiguiente para cada conjunto de medidas correspondiente a cada perspectiva se realizará esta Matriz de preferencia. La Matriz de Preferencia completa para los indicadores de la perspectiva financiera quedaría de la siguiente forma (Tabla 7).

Tabla 7 - Matriz de Preferencia - Medidas de Desempeño: Perspectiva Financiera

\begin{tabular}{|c|c|c|c|c|c|}
\hline Columnas & $\mathbf{J}$ & $\mathbf{j}$ & $\mathbf{j}$ & $\mathbf{J}$ & Filas \\
\hline & $\mathrm{MV}$ & $\mathrm{MN}$ & PC/CC & GA/UO & $\mathrm{i}$ \\
\hline Margen de Ventas (MV) & 1 & 5 & 3 & 5 & $\mathrm{i}$ \\
\hline Margen Neta (MN) & $1 / 5$ & 1 & 5 & 5 & $\mathrm{i}$ \\
\hline PC/CC & $1 / 3$ & $1 / 5$ & 1 & 3 & $\mathrm{i}$ \\
\hline GA/UO & $1 / 5$ & $1 / 5$ & $1 / 3$ & 1 & $\mathrm{i}$ \\
\hline Total & $13 / 4$ & $62 / 5$ & $91 / 3$ & 14 & \\
\hline
\end{tabular}

A partir de los datos presentados en la Matriz de Preferencia se procede a calcular los pesos y los valores relativos de importancia (VRI). Para hallar los pesos tomamos los valores de cada columna y los dividimos por su total, por ejemplo: $1 / 13 / 4=4 / 7=0,5714 \mathrm{y}$, así, para el resto de valores.

Los pesos calculados se colocan en una nueva matriz, la cual nos permite identificar los valores relativos de importancia con los cuales podemos establecer la jerarquía o importancia de cada medida, tal como se observa en la Tabla 8. 
LA INTEGRACIÓN DEL BALANCED SCORECARD (BSC) Y EL ANALYTIC HIERARCHY PROCESS (AHP) 100 PARA EFECTOS DE JERARQUIZAR MEDIDAS DE DESEMPEÑO Y TOMA DE DECISIÓN EN UNA INSTITUCIÓN FINANCIERA

Tabla 8 - Valores Relativos y Jerarquías de Importancia para Indicadores de la Perspectiva Financiera

\begin{tabular}{|c|c|c|c|c|c|c|}
\hline Indicadores & Pesos & Pesos & Pesos & Pesos & $\begin{array}{c}\text { Valores } \\
\text { Relativos de } \\
\text { Importancia } \\
\text { (VRI) }\end{array}$ & $\begin{array}{c}\text { Jerarquía de } \\
\text { Importancia }\end{array}$ \\
\hline & MV & MN & PC/CC & GA/UO & & \\
\hline $\begin{array}{c}\text { Margen de } \\
\text { Ventas (MV) }\end{array}$ & 0.57 & 0.78 & 0.31 & 0.36 & $\mathbf{0 . 5 0 5}$ & $\mathbf{1}$ \\
\hline $\begin{array}{c}\text { Margen Neta } \\
\text { (MN) }\end{array}$ & 0.12 & 0.16 & 0.54 & 0.36 & $\mathbf{0 . 2 9 5}$ & $\mathbf{2}$ \\
\hline PC/CC & 0.19 & 0.03 & 0.11 & 0.21 & $\mathbf{0 . 1 3 5}$ & $\mathbf{3}$ \\
\hline GA/UO & 0.12 & 0.03 & 0.04 & 0.07 & $\mathbf{0 . 0 6 5}$ & $\mathbf{4}$ \\
\hline Total & 1 & 1 & 1 & 1 & 1 & \\
\hline
\end{tabular}

Los valores que aparecen en esta Tabla 8 corresponden a los pesos que se obtienen a partir de los datos de la Matriz de Preferencia. La sumatoria de los pesos debe ser igual a 1 ó $100 \%$, cuando, expresado en porcentajes. Los VRIs son los valores resultantes del promedio de todos los pesos de una misma fila, por ejemplo:

$$
(0.57+0.78+0.31+0.36) / 4=0.505
$$

La jerarquía de importancia es designada de acuerdo al VRI. En este caso por ejemplo, el Margen de Ventas obtuvo el mayor VRI (0.505). Por lo tanto, su valor de jerarquía es uno (1), lo que significa su prioridad sobre el indicador GA/UO que obtuvo un VRI de 0,065 , equivalente a un valor en jerarquía de importancia de cuatro (dado que son 4 las medidas). Dicho valor significa que el indicador GA/UO es el menos prioritario de todos los indicadores analizados.

De esa manera, los resultados obtenidos en la Tabla 8 son el reflejo de los criterios que tuvieron los integrantes del grupo evaluador en el momento que se construyó la Matriz de Preferencia.

Este mismo análisis se realiza para el resto de indicadores que conforman las perspectivas de clientes, procesos internos, aprendizaje y crecimiento e, inclusive, se debe hacer para establecer el nivel de jerarquía entre las perspectivas, con lo cual se completa el proceso de jerarquización así, por ejemplo vide Tabla 9.

Tabla 9 - Matriz de Preferencia para las Perspectivas

\begin{tabular}{|c|c|c|c|c|}
\hline & PF & PC & PPI & PAYC \\
\hline Perspectiva Financiera (PF) & 1 & $1 / 3$ & 3 & $1 / 3$ \\
\hline Perspectiva de Clientes (PC) & 3 & 1 & 3 & 3 \\
\hline $\begin{array}{c}\text { Perspectiva de los Procesos Internos } \\
\text { (PPI) }\end{array}$ & $1 / 3$ & $1 / 3$ & 1 & $1 / 3$ \\
\hline $\begin{array}{c}\text { Perspectiva de Aprendizaje y } \\
\text { Crecimiento (PAYC) }\end{array}$ & 3 & $1 / 3$ & 3 & 1 \\
\hline Total & $71 / 3$ & 2 & 10 & $42 / 3$ \\
\hline
\end{tabular}

En el caso de las perspectivas observamos que según el criterio del grupo evaluador, la perspectiva más importante conforme los resultados obtenidos en la Tabla 10 es la de Clientes (nivel de jerarquía 1) y, la menos importante, es la perspectiva de los procesos internos (nivel de jerarquía 4). 
Tabla 10 - Valores Relativos de Importancia y Jerarquía para las Perspectivas

\begin{tabular}{|l|c|c|c|c|c|c|}
\hline \multicolumn{1}{|c|}{ Perspectivas } & Pesos & Pesos & Pesos & Pesos & $\begin{array}{c}\text { Valores } \\
\text { Relativos de } \\
\text { Importancia } \\
\text { VRI }\end{array}$ & $\begin{array}{c}\text { Jerarquía de } \\
\text { Importancia }\end{array}$ \\
\hline & PF & PC0 & PPI & PAYC & & .07 \\
\hline $\begin{array}{l}\text { Perspectiva Financiera } \\
\text { (PF) }\end{array}$ & .14 & .17 & .30 & $\mathbf{. 1 6 5 8 6 1 4 7 1 9}$ & $\mathbf{3}$ \\
\hline $\begin{array}{l}\text { Perspectiva de Clientes } \\
\text { (PC) }\end{array}$ & .41 & .50 & .30 & .64 & $\mathbf{. 4 6 2 9 8 7 0 1 3}$ & $\mathbf{1}$ \\
\hline $\begin{array}{l}\text { Perspectiva de } \\
\text { Procesos Internos (PPI) }\end{array}$ & .05 & .17 & .10 & .07 & $\mathbf{. 0 0 9 5 8 8 7 4 4 6}$ & $\mathbf{4}$ \\
\hline $\begin{array}{l}\text { Perspectiva de } \\
\text { Aprendizaje y } \\
\text { Crecimiento (PAYC) }\end{array}$ & .41 & .17 & .30 & .21 & $\mathbf{. 2 7 2 5 1 0 5 8 2 3}$ & $\mathbf{2}$ \\
\hline \multicolumn{1}{c}{ Total } & 1 & 1 & 1 & 1 & & $\mathbf{1}$ \\
\hline
\end{tabular}

\section{Paso 3. Calculo de los Índices de Desempeño Esperado y Real}

Una vez que se ha calculado el valor relativo en importancia y el nivel de jerarquías tanto para los indicadores por perspectiva como de las perspectivas, pasamos a calcular, el índice de desempeño esperado (IDE) y el índice de desempeño real (IDR) que cada medida debe tener. La importancia del IDE y del IDR radica en que nos permitirán monitorear el desempeño global.

Para calcular el IDE para cada indicador por perspectiva se procede de la siguiente forma: se toman los valores relativos de importancia (VRI $\mathrm{Indicador}_{\text {) }}$ obtenidos para cada conjunto de indicadores en cada perspectiva y los multiplicamos por cada valor relativo de importancia

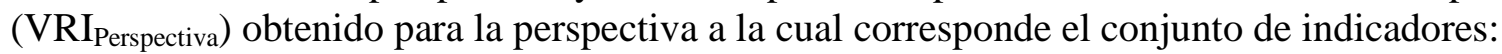

$$
\mathrm{IDE}_{\text {Indicador }}=\mathrm{VRI}_{\text {Indicador }} \mathrm{x} \quad \mathrm{VRI}_{\text {perspectiva }}
$$

La suma de los IDE $_{\text {indicador }}$ correspondientes a una misma perspectiva nos da el $\mathrm{IDE}_{\text {perspectiva: }}$

$$
\sum \mathrm{IDE}_{\text {Indicadores }}=\mathrm{IDE}_{\text {perspectiva. }}
$$

\begin{tabular}{|c|c|c|c|c|}
\hline Perspectiva Financiera & $\begin{array}{l}\text { Valor relativo de } \\
\text { Importancia de los } \\
\text { indicadores } \\
\text { VRI Indicador }^{\text {Indicas }}\end{array}$ & $\begin{array}{c}\text { Valor Relativo de } \\
\text { Importancia de la } \\
\text { perspectiva financiera } \\
\text { VRI }_{\text {perspectiva }}\end{array}$ & $\begin{array}{c}\text { Índice de } \\
\text { Desempeño } \\
\text { Esperado } \\
(\text { IDE) } \\
\text { VRI }_{\mathrm{I}} * \mathrm{VRI}_{\mathrm{P}}\end{array}$ & \\
\hline Margen de Ventas (MV) & 0.5091861326 & 0.168614719 & $8.59 \%$ & \\
\hline Margen Neta (MN) & 0.29112294 & 0.168614719 & $4.91 \%$ & \\
\hline Provis.Cartera/Cobrar & 0.136246566 & 0.168614719 & $2.30 \%$ & \\
\hline GA/UO & 0.00634444368 & 0.168614719 & $1.07 \%$ & \\
\hline Total & & & $16.86 \%$ & \\
\hline
\end{tabular}

Tabla 11 - Calculo de Indicadores de Desempeño Esperado para los Indicadores de la Perspectiva Financiera 


$$
\begin{gathered}
\sum\left(\mathrm{IDE}_{\text {Perspectiva Financiera }}+\mathrm{IDE}_{\text {Perspectiva Clientes }}+\mathrm{IDE}_{\text {Perspectiva Procesos Internos }}+\mathrm{IDE}_{\text {Perspectiva Aprendizaje y }}\right. \\
\text { Crecimiento }) \\
=\mathrm{IDE}_{\mathrm{Global}}
\end{gathered}
$$

$$
\mathrm{IDE}_{\text {Global }}=100 \%
$$

Una vez calculados los IDEs se procede al calcular los Índices de Desempeño Reales (IDRs). Los cuales se obtiene de la siguiente forma: se toma el valor Real obtenido para cada indicador durante el período y se divide entre el valor Meta fijado para el indicador. El resultado obtenido de la división anterior se multiplica con el IDE calculado para el indicador y, así, para cada indicador de cada perspectiva. La suma de los IDRs por cada perspectiva nos da el IDR global de la empresa.

$$
\begin{gathered}
\mathrm{IDR}_{\text {indicador }}=(\text { Valor Real del Indicador } / \text { Valor Meta del Indicador }) * \mathrm{IDE}_{\text {indicador }} \\
\sum \mathrm{IDR}_{\text {Indicadores de una misma persectiva }}=\mathrm{IDR}_{\text {Perspectiva }} \\
\sum\left(\mathrm{IDR}_{\text {Perspectiva Financiera }}+\mathrm{IDR}_{\text {Perspectiva Clientes }}+\mathrm{IDR}_{\text {Perspectiva Procesos Internos }}+\mathrm{IDR}_{\text {Perspectiva Aprendizaje y }}\right. \\
\text { Crecimiento }) \\
=\mathrm{IDR}_{\text {global }} \\
\mathrm{IDR}_{\text {Global }} \leq 0 ́ \mathbf{0} \geq 100 \%
\end{gathered}
$$

La importancia del calculo de los IDRs e IDEs radica en que al ser confrontados nos permiten identificar la perspectiva y los indicadores que deben ser mejorados en el siguiente período de tal forma que cada vez, la diferencia entre los índices de desempeño global (real y esperado) sea menor en un período.

El detectar que indicadores están por debajo de lo esperado lleva a la gerencia a tomar medidas correctivas, las cuales se concretizan en acciones, las cuales deben ser coherentes con las metas estratégicas de la compañía.

De esta manera, no se pierde de vista los aspectos relacionados con la implementación y ejecución de la estrategia en la organización y, adicionalmente, contamos con un sistema de monitoreo que no nos permite perder el rumbo, al alertarnos tempranamente de los desvíos.

Según lo anterior para el caso del Banco Social Colombiano S.A encontramos los siguientes IDEs e IDRs (Tabla 12). 
Tabla 12 - Índice de Desempeño Esperado e Índice de Desempeño Real Banco Social Colombiano S.A.

\begin{tabular}{|c|c|c|c|c|c|}
\hline $\begin{array}{c}\text { Medidas de Desempeño del Banco Social } \\
\text { Colombiano S.A }\end{array}$ & $\begin{array}{l}\text { AHP peso } \\
\text { o IDE }\end{array}$ & IDR & $\begin{array}{c}\text { Valor Real } \\
\text { del } \\
\text { Indicador }\end{array}$ & $\begin{array}{c}\text { Valor Meta } \\
\text { del } \\
\text { Indicador }\end{array}$ & $\begin{array}{c}\text { Variació } \\
\mathbf{n}\end{array}$ \\
\hline \multicolumn{6}{|l|}{ Perspectiva Financiera } \\
\hline 1. Margen de Ventas & $8.59 \%$ & $7.90 \%$ & $23 \%$ & $25 \%$ & $92 \%$ \\
\hline 2. Margen Neta & $4.91 \%$ & $4.58 \%$ & $14 \%$ & $15 \%$ & $93.33 \%$ \\
\hline 3: Prov.Cart/CC & $2.30 \%$ & $2.17 \%$ & $85 \%$ & $90 \%$ & $94.44 \%$ \\
\hline 4.GA/UO & $1.07 \%$ & $0.53 \%$ & $45 \%$ & $30 \%$ & $50 \%$ \\
\hline Total Perspectiva Financiera & $16.86 \%$ & $15.18 \%$ & & & \\
\hline \multicolumn{6}{|l|}{ Perspectiva Clientes } \\
\hline 5. No. De Prestamos Otorgados & $25.94 \%$ & $27.24 \%$ & $105 \%$ & 100 & $105 \%$ \\
\hline $\begin{array}{l}\text { 6. \% de Encuestas Contestadas } \\
\text { favorablemente }\end{array}$ & $14.43 \%$ & $12.83 \%$ & $80 \%$ & $90 \%$ & $88.88 \%$ \\
\hline 7. No. De errores cometidos & $5.92 \%$ & $-5.92 \%$ & 15 & 5 & $-100 \%$ \\
\hline Total Perspectiva Clientes & $46.30 \%$ & $34.14 \%$ & & & \\
\hline \multicolumn{6}{|l|}{ Perspectiva de los Procesos Internos } \\
\hline $\begin{array}{l}\text { Medidas de Desempeño del Banco Social } \\
\text { Colombiano S.A }\end{array}$ & $\begin{array}{l}\text { AHP peso } \\
\text { o IDE }\end{array}$ & IDR & $\begin{array}{c}\text { Valor Real } \\
\text { del } \\
\text { Indicador }\end{array}$ & $\begin{array}{c}\text { Valor Meta } \\
\text { del } \\
\text { Indicador }\end{array}$ & $\begin{array}{c}\text { Variació } \\
\mathbf{n}\end{array}$ \\
\hline 8. No. De Minutos en revisión SC & $2.07 \%$ & $2.23 \%$ & 55 & 60 & $8,33 \%$ \\
\hline 9. \% de clientes llamados por período & $3.46 \%$ & $2.31 \%$ & $60 \%$ & $90 \%$ & $66.66 \%$ \\
\hline 10. No. De nuevos servicios & $1.82 \%$ & $1.09 \%$ & 3 & 5 & $60 \%$ \\
\hline 11. No. De Políticas Implementadas / Período & $2.24 \%$ & $1.12 \%$ & 5 & 10 & $50 \%$ \\
\hline Total Perspectiva de los Procesos Internos & $9.59 \%$ & $6.41 \%$ & & & \\
\hline \multicolumn{6}{|l|}{ Perspectiva de Aprendizaje y Crecimiento } \\
\hline 12. $\%$ de Funcionarios Participantes & $18.12 \%$ & $19.26 \%$ & $85 \%$ & $80 \%$ & $106.25 \%$ \\
\hline 13. No. De Capacitaciones en el período & $6.30 \%$ & $1.89 \%$ & 3 & $10 \%$ & $30 \%$ \\
\hline 14. \% de Despidos por Período. & $2.83 \%$ & $4.53 \%$ & $2 \%$ & $5 \%$ & $40 \%$ \\
\hline $\begin{array}{l}\text { Total Perspectiva de Aprendizaje y } \\
\text { Crecimiento }\end{array}$ & $27.25 \%$ & $25.67 \%$ & & & \\
\hline $\begin{array}{l}\text { Índice de Desempeño Esperado y Real } \\
\text { Global }\end{array}$ & $100 \%$ & $81.78 \%$ & & & \\
\hline
\end{tabular}

\section{Paso 4. Análisis de los Índices de Desempeño Globales y por Indicador}

Tal como se observa en la Tabla 12, el desempeño de la empresa como un todo ha estado un poco por debajo del IDE global (100\%), una vez que el IDR global fue de $81,78 \% \%$. Los indicadores que tuvieron un buen desempeño por estar cerca de la meta fueron: Margen de Ventas (variación del 92\%), Margen Neta (variación del 93,33\%), PC/CC (variación 94,44\%), número de préstamos $(105 \%)$, número de minutos en revisión SC $(91,66 \%)$ y porcentaje de funcionarios participantes $(106,25 \%)$.

Por otro lado, los indicadores que estuvieron por debajo de la meta y que son los responsables de un IDR por debajo del $100 \%$ fueron: GA/U0 (variación 50\%), porcentaje de clientes llamados por período (variación 66,66\%), número de nuevos servicios (variación 60\%), número de políticas implementadas por período ( variación 50\%), número de capacitaciones en el período (variación 30\%), porcentaje de despidos por período (variación 40\%), número de errores cometidos (variación -100\%). 
De cara a los resultados obtenidos, los gerentes de área del Banco que obtuvieron indicadores por debajo o por encima (en el caso de aquellos que cuanto menor mejor) tendrán una mayor claridad para tomar medidas correctivas que mejoren los procesos que resultaron con Índices de Desempeño no tan buenos. De esta manera en el siguiente período y continuando con el proceso de monitoreo se podrá constatar sí las medidas correctivas produjeron los resultados esperados de mejoría tanto a nivel IDR global, como de cada indicador comprometido.

\section{CONCLUSIÓN}

El caso estudio del Banco Social Colombiano S.A ilustra como los indicadores identificados en el tablero de mando pueden ser integrados dentro de un sistema gerencial a través del uso del AHP de tal forma que un sistema de medidas de desempeño integral es constituido.

Esta metodología permitió que el Banco desarrollara mejor los objetivos estratégicos identificados para cada una de las perspectivas.

En el caso del Banco, el desempeño operacional se relaciona con las metas y las medidas de desempeño. Esta relación permite que la gerencia solo intervenga en los casos en los cuales los indicadores del tablero de mando han arrojado valores desfavorables y, por lo tanto, invitan a realizar revisiones para mejorar los indicadores conforme los cambios del mercado.

La ventaja de la metodología es que evita gastar tiempo y recursos en un conjunto de presupuestos y a realizar planes contingenciales que muchas veces no se relacionan con las estrategias plasmadas en un plan de largo plazo.

A nivel de los empleados también se ven los beneficios en la medida que para ellos queda más claro que es lo que crea valor para la empresa tanto en el corto como en el largo plazo y quienes son los responsables de que esto ocurra.

Finalmente, la integración del AHP y BSC da como resultado una metodología que invita a gerenciar para crear valor, usando el abordaje de indicadores en el desarrollo de la estrategia, por medio de la identificación de medidas y monitoreos continuos, construyendo buenos vínculos de comunicación entre la alta gerencia y los empleados de tal forma que el flujo de información entre los participantes se convierte en un factor critico de éxito.

\section{REFERENCIA}

BADDELEY, A. The magical number seven: still magic alter all these years. Psychological Review, p. 353-356, April, 1994. http://dx.doi.org/10.1037/0033-295X.101.2.353

CALATONE, R.J.; DI BENEDETTO, C.A.; SCHMIDT, J.B. Using the Analytic Hierarchy Process in new product screening. The Journal of Product Innovation Management, n. 16, p. 6576, 1999. http://dx.doi.org/10.1016/S0737-6782(98)00036-8

KAPLAN, R.; NORTON, D. Cuadro de Mando Integral. 2. ed. Barcelona: Gestión 2000, 1997.

KAPLAN, R.; NORTON, D. Mapas estratégicos. Barcelona: Gestión 2000, 2004.

MIN, H.; MITRA, A.; OSWALD, S. Competitive Benchmarking of health cara quality using the Analytic Hierarchy Process: an example from Korean Cancer Clinics. Socio-Economic Planning, v. 31, n. 2, p. 147-159, 1997. http://dx.doi.org/10.1016/S0038-0121(96)00021-3 
NIVEN, Paul. El cuadro de mando integral paso a paso. Barcelona: Gestión 2000, 2003.

REISINGER, H.; CRAVENS, K.; TELL, N. Prioritizing performance measures within the Balanced Scorecard framework. Management International Review. Fourth Quarter, p. 429-437, 2003.

SLATER, S.F.; OLSON, E.M.; REDDY, V.K. Strategy-based performance measurement. Business Horizons, p. 37-44, July/August, 1997. http://dx.doi.org/10.1016/S0007$\underline{6813(97) 90037-9}$

WU, Anne. The integration between Balanced Scorecard and Intellectual Capital. Journal of Intellectual Capital, p. 267-284, 2005. http://dx.doi.org/10.1108/14691930510592843 